\title{
Tabu Search directed by direct search methods for nonlinear global optimization *
}

\author{
Abdel-Rahman Hedar, Masao Fukushima* \\ Department of Applied Mathematics and Physics, Graduate School of Informatics, \\ Kyoto University, Kyoto 606-8501, Japan
}

\begin{abstract}
In recent years, there has been a great deal of interest in metaheuristics in the optimization community. Tabu Search (TS) represents a popular class of metaheuristics. However, compared with other metaheuristics like genetic algorithm and simulated annealing, contributions of TS that deals with continuous problems are still very limited. In this paper, we introduce a continuous TS called Directed Tabu Search (DTS) method. In the DTS method, direct-search-based strategies are used to direct a tabu search. These strategies are based on the well-known Nelder-Mead method and a new pattern search procedure called adaptive pattern search. Moreover, we introduce a new tabu list conception with anti-cycling rules called Tabu Regions and Semi-Tabu Regions. In addition, Diversification and Intensification search schemes are employed. Numerical results show that the proposed method is promising and produces high quality solutions.
\end{abstract}

Key words: Global optimization, Metaheuristics, Tabu search, Nelder-Mead method, Adaptive pattern direction

\section{Introduction}

Tabu Search (TS) is one of the recent metaheuristics originally developed for combinatorial optimization problems $[10,11]$. TS has shown an appropriate

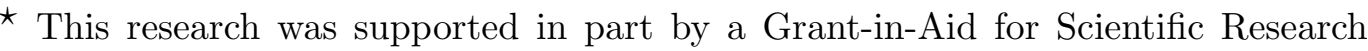
from the Ministry of Education, Science, Sports and Culture of Japan.

* Corresponding author.

Phone:+81 757535519

Fax: +81757534756

Email address: fuku@amp.i.kyoto-u.ac.jp (Masao Fukushima).
} 
performance when applied to these problems $[10,26]$. However, contributions of TS to solving continuous optimization problems are still very limited compared with other metaheuristics like Simulated Annealing and Genetic Algorithms. In this paper, we introduce a TS approach that deals with continuous global optimization problems. Specifically, we consider the unconstrained nonlinear minimization problem

$$
\min _{x \in R^{n}} f(x)
$$

where $f$ is a real-valued function defined on $R^{n}$. Recently, there has been a growing interest in solving this problem using metaheuristics $[25,26]$. However, metaheuristics may suffer from costly computations due to their slow convergence. So combining metaheuristics with local search methods is a practical remedy to overcome the slow convergence of metaheuristics. In particular, a number of attempts have been made to develop such combined search methods especially for Simulated Annealing and Genetic Algorithms [12-14]. In this paper, we present continuous versions of TS called Directed Tabu Search (DTS) by hybridizing TS with direct search methods. The role of direct search methods is to stabilize the search especially in the vicinity of a local minimum. Specifically, instead of using completely blind random search in generating neighborhood trial moves, appropriate direct search strategies are responsible to generate these neighborhood moves. Moreover, new implementations of TS elements are employed in the proposed method.

Since the first presentation of Glover [8,9], a lot of studies have emerged in the area of TS. The majority of these studies are related to combinatorial optimization problems and relatively few attempts have been made to deal with continuous optimization problems $[1-5,7,15]$. One of the earliest TS methods was presented by $\mathrm{Hu}$ [15] for constrained optimization problems. Cvijovic and Klinowski $[4,5]$ extented and generalized the TS to functions with variables that may be continuous or, if discrete, need not take integer values. Battiti and Tecchiolli [2] introduced an interesting continuous TS method called the Continuous Reactive Tabu Search. Their method tries to locate the most promising boxes, and then starting points for the local search are generated within those boxes. Al-Sultan and Al-Fawzan [1] gave a hybrid method that combines TS with the local search method of Hooke and Jeeves.

Recently, intensive studies on continuous TS have been conducted in [3,7]. In [3], the authors introduced a new algorithm called Enhanced Continuous Tabu Search (ECTS), which aims to follow Glover's basic approach as closely as possible. In order to cover a wide domain of possible solutions, the ECTS algorithm first performs a diversification search to locate the most promising areas. When the most promising areas are located, the algorithm proceeds to an intensification search within one promising area of the solution space. In 
[7], the authors presented a novel TS algorithm that explores a grid of points with a distance dynamically adjusted. When it collapses to a local minimum, it continues the local search from that point while accepting some non-improving points to allow the exploration of new regions in the search space.

The DTS method proposed in this paper differs from the previous continuous TS methods in many aspects. In the DTS method, three search procedures are employed; Exploration, Diversification and Intensification. In the Exploration Search, a new local search procedure is introduced to generate trial moves, based on the well-known Nelder-Mead method [24] and the recently proposed pattern search method [14]. Moreover, novel concepts of TS memory elements called Tabu Regions (TRs), Semi-TRs and a multi-ranked Tabu List (TL) are introduced to provide anti-cycling rules. Another memory element called Visited Regions List ( $V R L)$ is also introduced as a tool for the Diversification Search to diversify the search to unvisited areas of the solution space. Finally, assuming that one of the best points obtained by the Exploration and Diversification Searches is close to a global minimum, the Intensification Search is applied again at the final stage to refine the elite solutions visited so far. Actually, the proposed Diversification and Intensification Searches try to follow some known strategies from the high level TS with a long term memory. Moreover, the DTS can be classified as a multi-start method. The multi-start methods aim to construct powerful search procedures by guidance of global exploration and local searches; as surveys for multi-start methods the reader is referred to $[21,22,27]$. Multi-start methods have been successfully applied to both nonlinear global optimization and combinatorial problems, see [22] and references therein. Finally, the numerical results reported below show the promise of the proposed method especially in producing high quality solutions.

The rest of the paper is organized as follows. The next section gives a detailed description of the proposed TS memory elements. In Section 3, we introduce neighborhood and local search strategies used to generate the trial moves. The main DTS method is presented elaborately in Section 4. Section 5 discusses the implementation of the proposed method and reports comprehensive experimental results. The conclusion makes up Section 6 .

\section{TS Memory Elements}

The concept of memory plays a major role in TS, especially when it is used in a kind of learning process as in high level TS with a long term memory. Using an effective memory conception in intensification and diversification schemes makes TS behave as an intelligent search technique [10]. The optimization search methods can be classified in two categories; point-to-point methods and population-based methods. TS belongs to the first category. Keeping the 
diversity is one of the main problems that face the point-to-point methods compared with the population-based methods. However, the long term memory in TS makes it competitive with the population-based methods in keeping the diversity. In TS with long term memory, the search can be restarted from new diverse solutions whenever the diversification is needed, or can be intensified to improve the elite solutions whenever the intensification is needed. These TS concepts of diversification and intensification have turned out to be effective in many combinatorial optimization problems, see $[10,20]$ for example. In this section, we introduce some new conceptions and implementations of the TS memory elements to continuous optimization problems. First, we let the multi-ranked Tabu List $(T L)$ be a set of some visited solutions. The points in the $T L$ are ranked and saved according to their recency and their objective function values. Therefore, some positions in the $T L$ are kept for the best visited solutions, which helps an intensification scheme to refine the search from these best solutions at the final stage. Around each solution saved in the $T L$, two types of regions are specified in the search space. The first one is a Tabu Region $(T R)$ in which no new trial point is allowed to be generated. The other is a Semi-Tabu Region (Semi-TR) that comprises a surrounding region around $T R$. The main role of the Semi- $T R s$ is to generate neighboring trial points in a special way so that returning back to a visited $T R$ is avoided when the trial solution lies inside a Semi- TR. Another memory element introduced in this section is the Visited Region List ( $V R L)$. The centers of the visited regions and the frequency of visiting these regions are saved in the $V R L$ in order to direct a diversification scheme to explore the space outside these visited regions.

\subsection{Multi-Ranked Tabu List (TL)}

Some of the previously visited solutions are stored in the $T L$. Let $T L=\left\{t_{i}\right\}_{i=1}^{L}$. The elements in $T L$ are ranked in ascending order according to their recency using the rank indices $I_{i}^{r}, i=1, \ldots, L$, i.e., if the most recent element in $T L$ is $t_{k}$, then $I_{k}^{r}=1$, while if the most ancient element is $t_{k^{\prime}}$, then $I_{k^{\prime}}^{r}=L$. Also, the elements in $T L$ are ranked in ascending order according to their objective function values using another set of rank indices $I_{i}^{f v}, i=1, \ldots, L$, i.e., if the best element in $T L$ is $t_{j}$, then $I_{j}^{f v}=1$, and if the worst element is $t_{j^{\prime}}$, then $I_{j^{\prime}}^{f v}=L$. In the ordering, ties are broken arbitrarily. We consider the $T L$ to be a fuzzy set and associate its elements $\left\{t_{i}\right\}_{i=1}^{L}$ the membership values:

$$
m_{i}=\max \left\{m_{i}^{r}, m_{i}^{f v}\right\}, i=1, \ldots, L,
$$


where $m_{i}^{r}, m_{i}^{f v} \in[0,1]$ are the recency and the function-value ranked values, respectively, for element $t_{i}$ and they are computed as follows:

- The recency ranked value $m^{r}$. We use a linear ranking procedure that gives the most recent element the maximum ranked value $\eta_{\max }$ and the most ancient element the minimum ranked value $\eta_{\min }$, where $0 \leq \eta_{\min }<\eta_{\max } \leq 1$. Specifically, the recency ranked value for each element of $T L$ is given by

$$
m_{i}^{r}=\eta_{\min }+\left(\eta_{\max }-\eta_{\min }\right)\left(\frac{L-I_{i}^{r}}{L-1}\right), i=1, \ldots, L .
$$

- The function-value ranked value $m^{f v}$. To avoid reserving excessively many positions in the $T L$ for the best elements and to give the recency some priority, this procedure ranks only $\bar{L}$ best elements so that the best element is given the ranked value $\mu_{\max }$, and the worst $L-\bar{L}+1$ elements are given the ranked value $\mu_{\min }$, where $1 \leq \bar{L} \leq L$ and $0 \leq \mu_{\min }<\mu_{\max } \leq 1$. Specifically, the function-value ranked value for each element of $T L$ is given by

$$
m_{i}^{f v}= \begin{cases}\mu_{\min }+\left(\mu_{\max }-\mu_{\min }\right)\left(\frac{\bar{L}-I_{i}^{f v}}{\bar{L}-1}\right), & \text { if } I_{i}^{f v}=1, \ldots, \bar{L}, \\ \mu_{\min }, & \text { if } I_{i}^{f v}=\bar{L}+1, \ldots, L\end{cases}
$$

The Tabu Regions (TRs) are defined to be spheres with radius $r_{T R}$ and their centers being the points of $T L$, where $r_{T R}>0$. For each $T R$, we define Semi- $T R$ to be the surrounding region around this $T R$ with outer radius $r_{S T R}$ from its center, where $r_{S T R}>r_{T R}$. If a trial solution lies in Semi-TRs, then a specific procedure is applied to create special neighborhood trial points to avoid returning back to a vicinity of a previously visited solution. We suggest the following procedure for this purpose.

Procedure 2.1.

1. Let a trial point $x$ lie in $\nu$ Semi-TRs with centers $t_{1}, \ldots, t_{\nu}$. Compute the centroid $\bar{t}$ of the Semi-TRs' centers and the maximum distance $d_{\max }$ between $x$ and these centers, i.e.,

$$
\begin{aligned}
\bar{t} & =\frac{1}{\nu} \sum_{i=1}^{\nu} t_{i}, \\
d_{\max } & =\max _{i=1, \ldots, \nu}\left\{\left\|x-t_{i}\right\|\right\} .
\end{aligned}
$$

2. Construct neighborhood search directions that are parallel to the coordinate axes but point towards the direction $x-\bar{t}$, i.e., the neighborhood search directions are determined as $\operatorname{sign}\left((x)_{i}-(\bar{t})_{i}\right) e_{i}, i=1, \ldots, n$, where $e_{i} \in R^{n}$ is the $i$ th unit vector in $R^{n}$. Neighborhood trial points are generated along these search directions with a suitable step size $\beta>0$. In the case of $\nu>1$, the step size $\beta$ should be chosen greater than $d_{\max }+r_{T R}$ in order to avoid generating trial points inside a $T R$. 


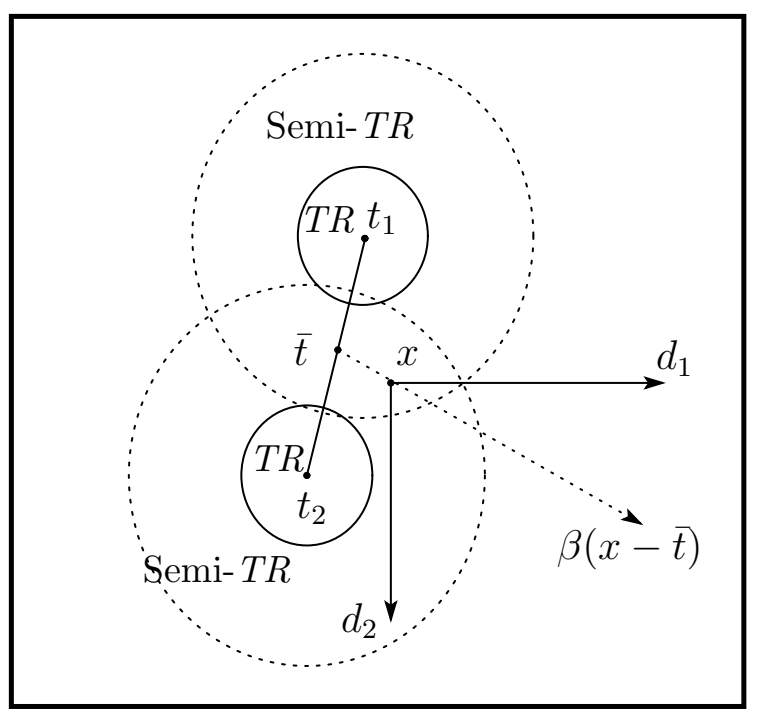

Fig. 1. Neighborhood search from a point in Semi-TRs.

Fig. 1 illustrates how Procedure 2.1 works when a solution $x$ lies in Semi$T R s$ in two dimensions. In this example, the solution $x$ lies in two Semi-TRs with center $t_{1}$ and $t_{2}$. According to Procedure 2.1, the neighborhood search directions $d_{1}$ and $d_{2}$ are constructed to follow the vector $(x-\bar{t})$, where $\bar{t}$ is the centroid of the Semi-TRs' centers. It is noteworthy that the step size used to generate a trial point along search directions $d_{1}$ and $d_{2}$ is chosen to be greater than $r_{T R}+\max \left\{\left\|x-t_{1}\right\|,\left\|x-t_{2}\right\|\right\}$, to make sure that the close TRs with centers $t_{1}$ and $t_{2}$ will not be hit.

\subsection{Visited Region List (VRL)}

Some historical information about the previously visited regions is stored in the $V R L$. More specifically, the center $\zeta_{i}$ of a visited region, which is a sphere with radius $\rho_{i}$, and the frequency $\varphi_{i}$ of visiting this region comprise the information stored in the $V R L$, i.e., $V R L=\left\{\left(\zeta_{i}, \rho_{i}, \varphi_{i}\right)\right\}_{i=1}^{M}$, where $M$ is the number of all listed visited regions. The information in $V R L$ will be used to direct the search towards new regions whenever the current TS procedure fails to get improvement or whenever a diversification scheme is needed. As a diversification scheme, we try to generate new trial points outside the visited regions. However, generating trial points near to more frequently visited regions is discouraged. To this end, a function $\Phi(\varphi)$ is introduced to distinguish between more and less frequently visited regions. Specifically, we define the function $\Phi$ as

$$
\Phi(\varphi)=\gamma\left(1-e^{-\gamma(\varphi-1)}\right)
$$


where $\gamma \in(0,1]$ is a given constant. Note that the function $\Phi$ is strictly increasing and bounded above by the value $\gamma$. We will describe the role of $\gamma$ in the diversification scheme after we state Procedure 2.2. below.

In the following, we suggest a procedure that uses the $V R L$ information to generate a new solution. The procedure allows accepting a trial point outside the visited regions, especially the more frequently visited ones.

\section{Procedure 2.2.}

1. Generate a trial point $x$ randomly in the search domain of $f$.

2. Compute the quantities $d_{i}=\left\|x-\zeta_{i}\right\| /\left(1+\Phi\left(\varphi_{i}\right)\right), i=1, \ldots, M$, where $\Phi(\varphi)$ is defined by (6). If $\min _{1 \leq i \leq M} d_{i} / \rho_{i} \geq 1$, then accept $x$. Otherwise, return to Step 1.

A point $x$ is accepted by Procedure 2.2 if it satisfies $\left\|x-\zeta_{i}\right\| / \rho_{i} \geq 1+\Phi\left(\varphi_{i}\right)$ for all $i=1, \ldots, M$. This means that no point can be accepted inside a previously visited region. Moreover, a point close to more frequently visited regions is hardly accepted. Therefore, the higher the value of $\gamma$ is, the lower the possibility of accepting a point close to more frequently visited regions is. To avoid infinitely cycling in Procedure 2.2, we may also terminate it after a predetermined number of iterations and return with $x$ corresponding to the maximum of the values of $\min _{1 \leq i \leq M} d_{i} / \rho_{i}$ over all iterations.

\section{$3 \quad$ Neighborhood-Local Search Strategies}

To explore the region around a solution and to generate the next move, we use neighborhood and local search strategies in which direct search methods are employed. Specifically, two search strategies are introduced to handle that job; Nelder-Mead Search (NMS) strategy and Adaptive Pattern Search (APS) strategy, which are based on the well-known Nelder-Mead method [24] and the recently proposed pattern search method [14], respectively. These neighborhood-local search strategies are invoked to generate trial points in the Exploration Search of the DTS method. More specifically, two types of trial points are generated by the neighborhood-local search strategy; neighborhood trial points and local trial points, which are needed in the Neighborhood Search and Local Search Steps, respectively, in Algorithm 4.1 stated in the next section. First, $p$ trial points $\left\{y_{i}\right\}_{i=1}^{p}$ are generated in a neighborhood of the current solution $x$. This procedure is called a neighborhood search, and the trial points $\left\{y_{i}\right\}_{i=1}^{p}$ are called neighborhood trial points. Then, we try to improve the neighborhood trial points $\left\{y_{i}\right\}_{i=1}^{p}$ by executing another search procedure, which is called a local search, to generate $q$ trial points $\left\{y_{p+i}\right\}_{i=1}^{q}$, which are called local trial points. The details of the neighborhood-local search strategies, NMS and APS, are given below. 


\subsection{Nelder-Mead Search (NMS) Strategy}

In the NMS strategy, we generate $p(=n)$ neighborhood trial points $\left\{y_{i}\right\}_{i=1}^{n}$, and $q(=1$ or 0$)$ local trial point. The neighborhood trial points are generated along search directions parallel to the coordinates axes starting from the current solution $x$ with suitable step sizes. If the current solution $x$ lies in a Semi- $T R$ or in Semi-TRs, we apply Procedure 2.1 to construct the search directions and the step sizes. Otherwise, we construct search directions parallel to the coordinate axes in a random way, i.e., each of them is parallel to a positive or a negative coordinate direction. To generate a local trial point, we construct a simplex $S$ that consists of the current solution $x$ and the current $n$ neighborhood trial points $\left\{y_{i}\right\}_{i=1}^{n}$, i.e., $S=\left\{x, y_{1}, \ldots, y_{n}\right\}$. Some iterations of the NM method are applied starting from $S$. If an improvement point is obtained from these NM iterations, then we set the local trial point $y_{n+1}$ equal to this improvement point, i.e., $q=1$. Otherwise, there is no trial point, i.e., $q=0$.

For more explanation of the NMS strategy, we show an example in two dimensions in Fig. 2. Given the current solution $x$, two neighborhood trial points $y_{1}$ and $y_{2}$ are generated in a neighborhood of $x$ as in Fig. 2(a). To find a local trial point, we construct a simplex whose vertices are $S=\left\{x, y_{1}, y_{2}\right\}$, as in Fig. 2(b). Assuming that the worst point in $S$ is $y_{2}$, we apply the Nelder-Mead method operations described in Fig. 2(c) to find a better movement. If one exists, we refer to this better movement as a local trial point.

\subsection{Adaptive Pattern Search (APS) Strategy}

The main idea behind the APS strategy is based on the approximate descent direction (ADD) method proposed in [14]. The ADD method is a derivativefree procedure with high ability of producing a descent direction. In the ADD method, several points around a given point $x \in R^{n}$ are used to generate an approximate descent direction of function $f$ at $x$. We implement the same procedure as in [14] to produce a new adaptive direction from standard pattern directions. Specifically, we construct $n$ pattern directions parallel to the coordinate axes emanating from the point $x$ and generate $n$ trial points $\left\{y_{i}\right\}_{i=1}^{n}$ along these directions with a suitable step size. The adaptive direction $v$, along which we may expect to decrease the function value, is computed using these trial points as follows:

$$
v=\sum_{i=1}^{n} \omega_{i} u_{i}
$$




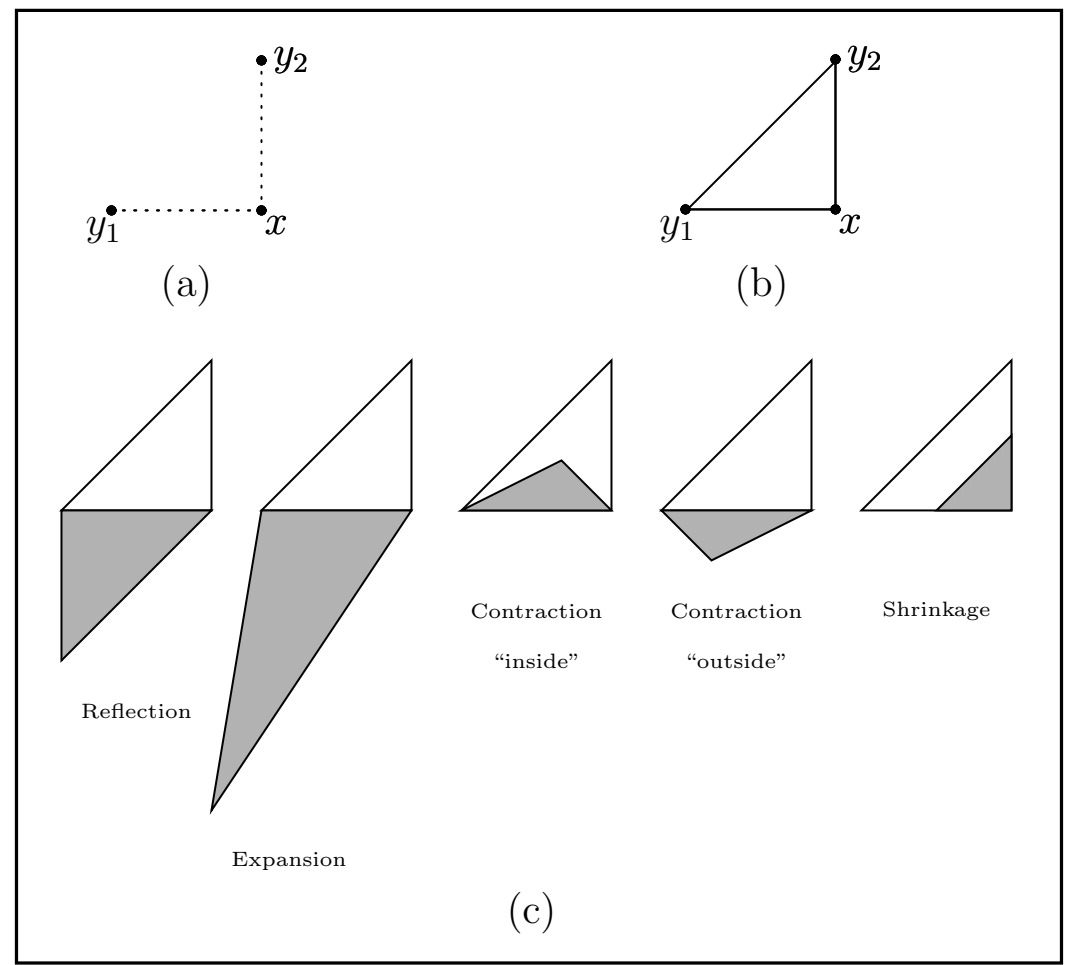

Fig. 2. NMS strategy in two dimensions.

where

$$
\begin{gathered}
\omega_{i}=\frac{\Delta f_{i}}{\sum_{j=1}^{n}\left|\Delta f_{j}\right|}, \quad i=1,2, \ldots, n, \\
u_{i}=-\frac{\left(y_{i}-x\right)}{\left\|y_{i}-x\right\|}, \quad i=1,2, \ldots, n, \\
\Delta f_{i}=f\left(y_{i}\right)-f(x), \quad i=1,2, \ldots, n .
\end{gathered}
$$

In the APS strategy, we generate $p(=n)$ neighborhood trial points $\left\{y_{i}\right\}_{i=1}^{n}$ using the standard pattern directions, and $q(=2)$ local trial points using an adaptive pattern direction. More specifically, we construct $n$ pattern directions parallel to the coordinate axes emanating from the current solution $x$ and generate $n$ neighborhood trial points $\left\{y_{i}\right\}_{i=1}^{n}$ along these directions with some step size. The adaptive pattern direction $v$ at $x$ is computed using (7). Two local trial points $y_{n+1}$ and $y_{n+2}$ are generated along the vector $v$ with two different step sizes.

An example in two dimensions is illustrated in Fig. 3 to describe the APS strategy. Two neighborhood trial points $y_{1}$ and $y_{2}$ are generated in a neighborhood of the current solution $x$ as in Fig. 3(a). An approximate descent direction $v$ is computed at $x$ using the points $y_{1}$ and $y_{2}$, as in (7). If we assume that $x$ is better than $y_{1}$ and $y_{2}$, then, by means of (7), the vector $v$ is 


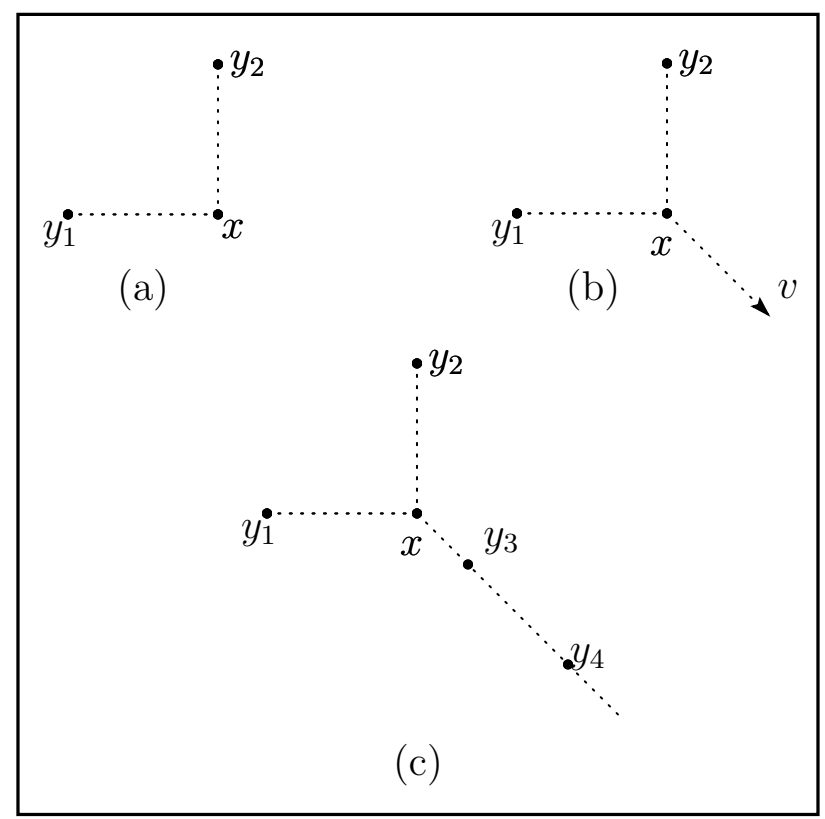

Fig. 3. APS strategy in two dimensions.

composed toward the vectors $x-y_{1}$ and $x-y_{2}$ with weights inversely proportional to $\left|f(x)-f\left(y_{1}\right)\right|$ and $\left|f(x)-f\left(y_{2}\right)\right|$, see Fig. 3(b). Finally, two local trial points $y_{3}$ and $y_{4}$ are generated along the vector $v$ with two different step sizes in order to explore the area along the promising direction $v$ as in Fig. 3(c).

\section{Directed Tabu Search (DTS)}

In this section, we describe some details about how a TS method is modified with the memory elements and neighborhood-local search strategies introduced in Sections 2 and 3 to compose the DTS method.

In the DTS method, three basic search procedure are used; Exploration, Diversification and Intensification search procedures. In the Exploration Search, we use the neighborhood-local search strategies, which are described in Section 3, to explore the solution space. Moreover, the multi-ranked TL, TR and Semi- $T R$ restriction rules are applied to avoid revisiting recently visited solutions or being entrapped in local minima. Then, the Diversification Search is needed in order to diversify the search to other areas of the solution space that may have been overlooked in the Exploration Search. We use the $V R L$ and Procedure 2.2 to manage the Diversification Search. Finally, in order to explore the close regions around the best points visited so far, the Intensification Search is applied to refine these best points. These search procedures are applied in such a way that they give the DTS method a better chance to explore the search space efficiently. Actually, the Exploration and Diversifica- 


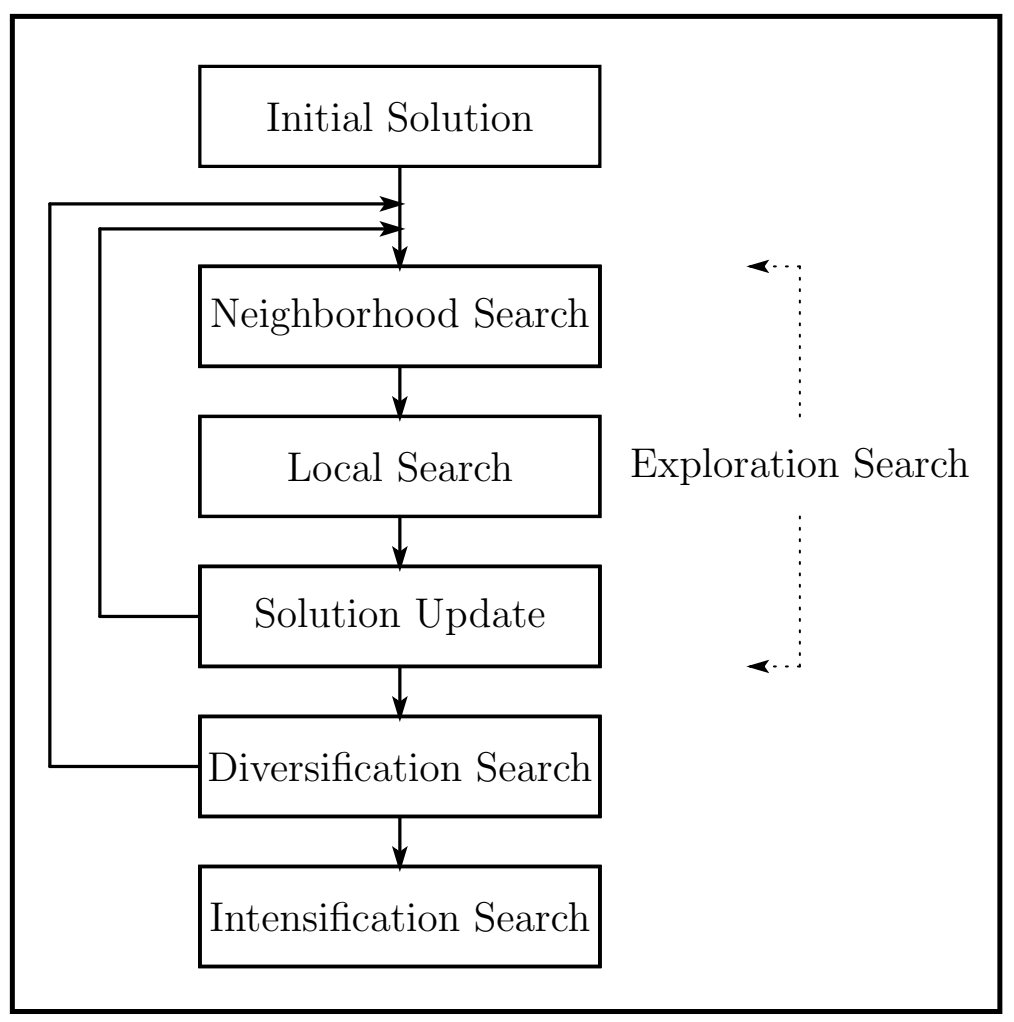

Fig. 4. Main structure of the DTS method.

tion search procedures are assembled to compose the DTS main loop and are repeated until the termination conditions are satisfied. Moreover, the Exploration Search procedure is included as an inner loop within the diversification loop. We will use the superscript $j=0,1, \ldots$, to represent the main loop iteration counter, the subscript $k=0,1, \ldots$, to represent the inner loop iteration counter, and $x_{k}^{(j)}$ to denote a general iterate. In other words, the Exploration and Diversification search procedures compose a multi-start procedure with a long term memory. At the final stage, the Intensification Search procedure based on elite TS is needed to complete the DTS method. The main structure of the DTS method is shown in Fig. 4. More detailed description of the search procedures is given below.

\subsection{Exploration-Diversification Loop}

The main loop of the DTS method, which consists of Exploration and Diversification Searches, starts with an initial solution $x_{0}^{(0)}$. In each main loop iteration $j$, the Exploration Search procedure is repeatedly applied to obtain improvement by means of neighborhood-local search strategies, and then the Diversification Search procedure is applied to locate a new initial trial point $x_{0}^{(j+1)}$, from which the Exploration Search is restarted again. This main loop is repeated at most $\ell_{\text {main }}$ times, where $\ell_{\text {main }}$ is a predetermined positive integer. 
Exploration Search. The Exploration Search starts with an initial solution $x_{0}^{(j)}$ at each main loop iteration $j$. In each iteration of the Exploration Search, a neighborhood-local search (NMS or APS) strategy is used to generate $n$ neighborhood trial points $\left\{y_{i}\right\}_{i=1}^{n}$ in a neighborhood of the current iterate $x_{k}^{(j)}$. If a better movement is found among these trial points, we update the current iterate and proceed to the next inner loop iteration. Otherwise, i.e., $x_{k}^{(j)}$ is still better than all neighborhood trial points, the neighborhood-local search strategy continues to generate $q$ local trial points $\left\{y_{n+i}\right\}_{i=1}^{q}$, where $q=0$ or 1 in the NMS strategy and $q=2$ in the APS strategy. Then, the current iterate $x_{k}^{(j)}$ is updated to be the best of neighborhood and local trial points, i.e., $x_{k+1}^{(j)}:=\arg \min _{i=1, \ldots, n+q}\left\{f\left(y_{i}\right)\right\}$. TL is also updated by letting $x_{k}^{(j)}$ replace the element with the smallest membership value. If a new region is reached, then $V R L$ should be updated by adding the information on this region. This Exploration Search loop is repeated at most $\ell_{\text {inner }}$ times, where $\ell_{\text {inner }}$ is a predetermined positive integer.

Diversification Search. The Diversification Search is carried out when the Exploration Search either spends the inner iterations $\ell_{\text {inner }}$ times or fails to obtain an improvement in some consecutive iterations. With the current $V R L$, Procedure 2.2 is applied to generate a trial point $x_{0}^{(j+1)}$ in some new region. Then the Exploration Search is repeated again starting from $x_{0}^{(j+1)}$.

\subsection{Intensification Search}

According to the principle of the multi-ranked $T L$, it reserves the best points visited so far. In order to improve these points, we complete the DTS method by applying another local search method starting from some of these points, which we call Intensification Search. We use Kelley's modification $[16,17]$ of the Nelder-Mead (NM) method as a local search method for this task.

\subsection{Main Algorithm}

We have two versions of the DTS method; $\mathrm{DTS}_{N M S}$ and $\mathrm{DTS}_{A P S}$ that use the NMS strategy and the APS strategy, respectively, as neighborhood-local search strategies. First, a specific and formal description of $\mathrm{DTS}_{N M S}$ is given in the following Algorithms 4.1.

Algorithm 4.1. $\operatorname{DTS}_{N M S}\left(f, x_{0}^{(0)}\right)$

1. Initialization. Choose positive integers $\ell_{\text {main }}, \ell_{\text {main }}^{\prime}, \ell_{\text {inner }}$ and $\ell_{\text {inner }}^{\prime}$. Choose an initial solution $x_{0}^{(0)}$, and set $T L$ and $V R L$ to be empty.

2. Exploration-Diversification Search (Main Loop). Let $j:=0$ and 
repeat this main loop until $\ell_{\text {main }}^{\prime}$ consecutive main iterations fail to obtain improvement or the main loop iteration counter $j$ exceeds $\ell_{\text {main }}$.

2.1. Exploration Search(NMS) (Inner Loop). Let $k:=0$ and repeat this inner loop until $\ell_{\text {inner }}^{\prime}$ consecutive inner iterations fail to obtain improvement or the inner loop iteration counter $k$ exceeds $\ell_{i n n e r}$.

2.1.1. Search Directions. If the current iterate $x_{k}^{(j)}$ lies in Semi-TRs, use Procedure 2.1 to construct search directions $\left\{d_{i}\right\}_{i=1}^{n}$ and to choose step sizes $\left\{\Delta_{i}\right\}_{i=1}^{n}$. Otherwise, construct search directions $d_{i}:=(-1)^{\tau_{i}} e_{i}, i=1, \ldots, n$, where $e_{i} \in R^{n}$ is the $i$ th unit vector in $R^{n}$ and $\tau_{i}$ is a random integer number, and choose suitable step sizes $\left\{\Delta_{i}\right\}_{i=1}^{n}$.

2.1.2. Neighborhood Search. Generate $n$ neighborhood trial points $y_{i}:=$ $x_{k}^{(j)}+\Delta_{i} d_{i}, i=1, \ldots, n$. Whenever a better movement is found during this process, stop generating points, set $x_{k+1}^{(j)}$ equal to this better movement, and go to Step 2.1.4.

2.1.3. Local Search. Apply $n$ iterations of the NM method starting from the simplex $S:=\left\{x_{k}^{(j)}, y_{1}, \ldots, y_{n}\right\}$. If an improvement point is obtained from these NM iterations, set local trial point $y_{n+1}$ equal to this improvement point, and set $q:=1$. Otherwise, set $q:=0$. Set $x_{k+1}^{(j)}:=\arg \min _{i=1, \ldots, n+q}\left\{f\left(y_{i}\right)\right\}$.

2.1.4. Parameter Update. Let $x_{k}^{(j)}$ replace the element with the smallest membership value in $T L$ and re-rank the $T L$ elements using (1). Update the $V R T$ and set $k:=k+1$.

2.2. Diversification Search. Generate a trial point $x_{0}^{(j+1)}$ using Procedure 2.2. Update the $T L$ and $V R T$, and set $j:=j+1$.

3. Intensification Search. Apply the Kelley's modification [16] of the NM method starting from some elite solutions in the $T L$.

The DTS $A P S$ algorithm is the same as Algorithm 4.1 except Step 2.1, which should be changed as follows:

2.1. Exploration Search(APS) (Inner Loop). Let $k:=0$, initialize a vector $v$ to be a random vector in $R^{n}$, and repeat this inner loop until $\ell_{\text {inner }}^{\prime}$ consecutive inner iterations fail to obtain improvement or the inner loop iteration counter $k$ exceeds $\ell_{\text {inner }}$.

2.1.1. Search Directions. If the current iterate $x_{k}^{(j)}$ lies in Semi-TRs, use Procedure 2.1 to construct search directions $\left\{d_{i}\right\}_{i=1}^{n}$ and to choose step sizes $\left\{\Delta_{i}\right\}_{i=1}^{n}$. Otherwise, construct search directions $d_{i}:=\operatorname{sign}\left(v_{i}\right) e_{i}, i=$ $1, \ldots, n$, where $e_{i} \in R^{n}$ is the $i$ th unit vector in $R^{n}$ and $v_{i}$ is the $i$ th component of $v$, and choose suitable step sizes $\left\{\Delta_{i}\right\}_{i=1}^{n}$.

2.1.2. Neighborhood Search. Generate $n$ neighborhood trial points $y_{i}:=$ $x_{k}^{(j)}+\Delta_{i} d_{i}, i=1, \ldots, n$. Whenever a better movement is found during this process, stop generating points, set $x_{k+1}^{(j)}$ equal to this better movement, and go to Step 2.1.4.

2.1.3. Local Search. Compute the direction $v$ at $x_{k}^{(j)}$ using $\left\{y_{i}\right\}_{i=1}^{n}$ as in (7). Choose two suitable step sizes $\alpha_{1}$ and $\alpha_{2}$ to generate local trial points 
$y_{n+i}=x_{k}^{(j)}+\alpha_{i} v /\|v\|, i=1,2$. Set $x_{k+1}^{(j)}:=\arg \min _{i=1, \ldots, n+2}\left\{f\left(y_{i}\right)\right\}$.

2.1.4. Parameter Update. Let $x_{k}^{(j)}$ replace the element with the smallest membership value in $T L$ and re-rank the $T L$ elements using (1). Update the $V R T$ and set $k:=k+1$.

\section{Implementation and Experiments}

In this section, we give more details about the implementation as well as the experimental results of the DTS algorithms.

\subsection{Setting Parameters}

In this subsection, we discuss the suggested values of the parameters needed in the implementation of the DTS algorithms and the sensitivity of these parameters. First, the initial trial solution $x_{0}^{(0)}$ is chosen randomly from the predetermined range $[\mathfrak{L}, \mathfrak{U}]$ of the initial points for each test function, where $[\mathfrak{L}, \mathfrak{U}]:=\left\{x \in R^{n}: l_{i} \leq x_{i} \leq u_{i}, i=1, \ldots, n\right\}$. The other parameters can be classified into the following groups.

- $\boldsymbol{T R}$ and Semi- $\boldsymbol{T R}$ parameters: the radius $r_{T R}$ of each $T R$, and the outer radius $r_{S T R}$ of the Semi- $T R$.

- $\boldsymbol{T L}$ parameters: the number $L$ of elements in $T L$, the maximum and minimum recency ranked values $\eta_{\max }$ and $\eta_{\min }$, respectively, the number $\bar{L}$ of the function-value ranked elements, and the maximum and minimum function-value ranked values $\mu_{\max }$ and $\mu_{\min }$, respectively.

- $\boldsymbol{V R} \boldsymbol{L}$ parameters: the radii $\rho_{j}, j=1, \ldots, M$, of the visited regions.

- Step sizes: the step sizes $\Delta_{i}, i=1, \ldots, n$, used in generating neighborhood trial points in $\mathrm{DTS}_{N M S}$ and $\mathrm{DTS}_{A P S}$, and $\alpha_{1}$ and $\alpha_{2}$ used to generate local trial points in $\mathrm{DTS}_{A P S}$.

- Diversification trials: the parameter $\gamma$ used in (6) and the maximum number $I t_{\max }$ of iterations allowed in Procedure 2.2.

- Intensification trials: the number $N_{\text {best }}$ of best points that are used in the Intensification Search.

- Termination conditions: the loop termination numbers $\ell_{\text {main }}, \ell_{\text {main }}^{\prime}, \ell_{\text {inner }}$ and $\ell_{i n n e r}^{\prime}$.

Proper values of these parameters have been studied through extensive numerical experiments by using the functions Branin $(R C)$, Goldstein\&Price $(G P)$, Rosenbrock $\left(R_{2}\right)$ and Zakhrov $\left(Z_{2}\right)$ and $\left(Z_{5}\right)$. In the tuning parameters experiments, we have tried to find a standard setting of the DTS parameters which is problem-independent as much as possible. Moreover, some relations between 
the parameters have been discussed to guide the user to set the DTS parameters whenever new implementations of the DTS algorithm are invoked. Below, we highlight the conclusion we got from the tuning parameters experiments.

First, the values of the parameter $\gamma$ that we have studied are $0.10,0.15,0.2, \ldots$, 0.4. Recall that the main role of this parameter is to avoid generating a new diverse trial solution near to the more frequently visited regions. Since large $\gamma$ may lead to a big area surrounding the more frequently visited regions left without exploration, we did not test a value of $\gamma$ higher than 0.4 . The performance of the DTS algorithms is almost the same for all runs using the above-mentioned values of $\gamma$. Moreover, at the end of running the DTS algorithms on many test functions, the centers of the visited regions listed in the $V R L$ are distributed almost uniformly for all tested values of $\gamma$. However, the value $\gamma=0.25$ produces slightly more scattered distributions than the other values. Therefore, we set $\gamma$ equal to 0.25 . The parameter $I t_{\max }$ is set equal to $100 n$.

Most of the DTS parameters listed above are distance parameters. These distance parameters are $r_{T R}, r_{S T R}, \rho, \Delta_{i}, i=1, \ldots, n, \alpha_{1}$ and $\alpha_{2}$. Note that the radii $\rho_{j}, j=1, \ldots, M$, of all visited regions are set equal to $\rho$. For more accurate setting of the values of these distance parameters, we consider the following:

(1) Since Semi-TRs are surrounding TRs, we let $r_{S T R}>r_{T R}$. For easily escaping from TRs and Semi-TRs, it is desirable to let $\Delta_{i}>r_{S T R}$. Moreover, to avoid producing too many small visited regions, we let $\rho>\Delta_{i}$. This means that the desirable order of the distance parameters is $r_{T R}<r_{S T R}<\Delta_{i}<\rho$. Since the step sizes $\alpha_{1}$ and $\alpha_{2}$, are used to search the area along an approximate descent direction, it is appropriate to let one of them be smaller than the usual step size $\Delta_{i}$ and the other be greater than $\Delta_{i}$.

(2) To keep the distance parameters in the above order, we let their values relate to only one parameter $\delta$ which is the diameter of the range $[\mathfrak{L}, \mathfrak{U}]$ defined as $\delta:=\max _{1 \leq i \leq n}\left(u_{i}-l_{i}\right)$.

The performance of the DTS algorithms were tested using different values for these parameters through many test functions. The suggested values of these parameters are given in Table 1 , and $r_{S T R}$ is set equal to $2 r_{T R}$. The performance of the DTS algorithms were almost insensitive with regard to all tested values of the distance parameters. In Table 1, we also suggest the value for each parameter which produces the best performance. For more efficient search, the step sizes may be randomly chosen close to some fixed mean values, rather than being set at fixed values. Specifically, we set the step sizes as follows: 
Table 1

Distance parameters.

\begin{tabular}{ccc}
\hline Parameter & Tested values & Suggested value \\
\hline$r_{T R}$ & $0.01 \delta, 0.02 \delta, 0.03 \delta, 0.04 \delta$ & $0.01 \delta$ \\
$\Delta_{i}$ & $0.08 \delta, 0.1 \delta, 0.12 \delta$ & $0.1 \delta$ \\
$\rho$ & $0.15 \delta, 0.2 \delta, 0.25 \delta$ & $0.15 \delta$ \\
\hline
\end{tabular}

$$
\begin{aligned}
& \Delta_{i}=\left(0.1+0.025 \omega_{i}\right) \delta, i=1, \ldots, n, \\
& \alpha_{1}=\left(0.1-0.05 \theta_{1}\right) \delta \\
& \alpha_{2}=\left(0.1+0.05 \theta_{2}\right) \delta
\end{aligned}
$$

where $\omega_{i}, i=1, \ldots, n$, are random numbers from the interval $(-1,1)$, and $\theta_{1}$ and $\theta_{2}$ are random numbers from the interval $(0,1)$.

For the $T L$ parameters, the values $5 n, 6 n, 7 n$ and $8 n$ and the values $2 n, 3 n$ and $4 n$ have been tested as possible choices for $L$ and $\bar{L}$, respectively. The performance of the DTS algorithms using these values of $L$ and $\bar{L}$ is almost the same. So, to avoid storing unnecessary information, we set $L$ and $\bar{L}$ equal to the least possible values, i.e., $L=5 n$ and $\bar{L}=2 n$. The other $T L$ parameters are set as $\eta_{\max }=\mu_{\max }=1$ and $\eta_{\min }=\mu_{\min }=1 / L$.

The parameter $N_{\text {best }}$ is set equal to 1 because the numerical results show that the best point found in the Exploration-Diversification Search is close to global minima for most of the test function.

The last group of parameters are related to the termination conditions. Actually, choosing sufficiently large values for the loop termination numbers $\ell_{\text {main }}$, $\ell_{\text {main }}^{\prime}, \ell_{\text {inner }}$ and $\ell_{\text {inner }}^{\prime}$ is highly needed to avoid premature termination of the method. The numerical results have shown that the lowest values of these parameters that can give an acceptable performance of the DTS algorithms are $\ell_{\text {main }}=\ell_{\text {inner }}=5 n$, and $\ell_{\text {main }}^{\prime}=\ell_{\text {inner }}^{\prime}=2 n$. However, higher values for these numbers can increase the ability of finding global minima for some difficult test problems.

\subsection{Numerical Results}

In this subsection, we discuss the performance of the DTS algorithms through two main experiments. The first experiment is to compare the results obtained by the $\mathrm{DTS}_{N M S}$ and DTS $A P S$ and then compare the best version of them with other continuous versions of TS. In the second experiment, the performance of the best DTS algorithms is also compared with other metaheuristics. 
Table 2

Test functions (Set A).

\begin{tabular}{llllllll}
\hline No. & $f$ & Function name & $n$ & No. & $f$ & Function name & $n$ \\
\hline 1 & $R C$ & Branin RCOS & 2 & 9 & $S_{4,5}$ & Shekel & 4 \\
2 & $E S$ & Easom & 2 & 10 & $S_{4,7}$ & Shekel & 4 \\
3 & $G P$ & Goldstein\&Price & 2 & 11 & $S_{10,7}$ & Shekel & 4 \\
4 & $S H$ & Shubert & 2 & 12 & $Z_{5}$ & Zakharov & 5 \\
5 & $Z_{2}$ & Zakharov & 2 & 13 & $R_{5}$ & Rosenbrock & 5 \\
6 & $R_{2}$ & Rosenbrock & 2 & 14 & $H_{6,4}$ & Hartmann & 6 \\
7 & $D J$ & De Joung & 3 & 15 & $Z_{10}$ & Zakharov & 10 \\
8 & $H_{3,4}$ & Hartmann & 3 & 16 & $R_{10}$ & Rosenbrock & 10 \\
\hline
\end{tabular}

\subsubsection{Numerical Results of DTS and other TS methods}

To examine the performance of the DTS algorithms DTS $_{N M S}$ and DTS $A P S$, we tested them on some well known functions [6,13] listed as Set A in Table 2. The characteristics of these test functions are diverse enough to cover many kinds of difficulties that arise in global optimization problems. To complete the evaluation of the DTS algorithms, they should be compared with other continuous versions of TS. However, it is not easy to show complete and fair comparisons due to the lack of some information especially on the quality of solutions obtained by those continuous TS methods. Therefore, we try to compare our algorithms with other continuous TS methods in terms of the ability of obtaining global minima, the cost of function evaluations and the quality of computed solutions. Three continuous TS methods chosen to compare with the DTS algorithms are continuous reactive TS (CRTS) [2], Enhanced Continuous Tabu Search (ECTS) [3], and TS-based algorithm called DOPE [7]. The ECTS and DOPE methods are the most recent continuous TS methods and the quality of computed solutions are stated clearly in their original papers.

For each function in Set A, we applied the DTS codes 100 times with different starting points. For all these test functions, we used the same values of the DTS parameters as those presented in Subsection 5.1. Moreover, we used the same condition as that used by ECTS [3] to judge the success of a trial, which is given by

$$
\left|f^{*}-\tilde{f}\right|<\epsilon_{1}\left|f^{*}\right|+\epsilon_{2}
$$

where $\tilde{f}$ refers to the best function value obtained by the algorithm, $f^{*}$ refers to the exact global minimum, and $\epsilon_{1}$ and $\epsilon_{2}$ are set equal to $10^{-4}$ and $10^{-6}$, respectively. Note that the conditions for successful trials are not stated for 
Table 3

Results of DTS algorithms.

\begin{tabular}{|c|c|c|c|c|c|c|}
\hline \multirow[b]{3}{*}{$f$} & \multicolumn{3}{|c|}{$\mathrm{DTS}_{N M S}$} & \multicolumn{3}{|c|}{$\operatorname{DTS}_{A P S}$} \\
\hline & f-evals. & & & f-evals. & & \\
\hline & Av. $(\min / \max )$ & $A v . E r$ & Suc. & $A v \cdot(\min / \max )$ & $A v . E r$ & Suc. \\
\hline$R C$ & $274(252 / 296)$ & $4 \mathrm{e}-7$ & $100 \%$ & $212(181 / 243)$ & $4 \mathrm{e}-7$ & $100 \%$ \\
\hline$E S$ & $271(202 / 285)$ & $5 \mathrm{e}-9$ & $30 \%$ & $223(156 / 244)$ & $4 \mathrm{e}-9$ & $82 \%$ \\
\hline$G P$ & $293(276 / 324)$ & $5 \mathrm{e}-9$ & $88 \%$ & $230(207 / 282)$ & $5 e-9$ & $100 \%$ \\
\hline$S H$ & $298(282 / 319)$ & $9 \mathrm{e}-6$ & $44 \%$ & $274(260 / 307)$ & $9 \mathrm{e}-6$ & $92 \%$ \\
\hline$Z_{2}$ & $273(247 / 291)$ & $6 \mathrm{e}-9$ & $100 \%$ & $201(183 / 225)$ & $5 \mathrm{e}-9$ & $100 \%$ \\
\hline$R_{2}$ & $358(272 / 489)$ & $6 e-9$ & $100 \%$ & $254(207 / 321)$ & $5 e^{-9}$ & $100 \%$ \\
\hline$D J$ & $650(600 / 694)$ & $5 \mathrm{e}-9$ & $100 \%$ & $446(393 / 516)$ & $4 \mathrm{e}-9$ & $100 \%$ \\
\hline$H_{3,4}$ & $670(613 / 789)$ & $2 \mathrm{e}-6$ & $97 \%$ & $438(389 / 493)$ & $2 \mathrm{e}-6$ & $100 \%$ \\
\hline$S_{4,5}$ & $1426(1342 / 1473)$ & $7 \mathrm{e}-7$ & $39 \%$ & $819(669 / 989)$ & $3 \mathrm{e}-7$ & $75 \%$ \\
\hline$S_{4,7}$ & $1425(1372 / 1487)$ & $4 \mathrm{e}-5$ & $29 \%$ & $812(675 / 973)$ & $4 \mathrm{e}-5$ & $65 \%$ \\
\hline$S_{4,10}$ & $1438(1340 / 1493)$ & $1 \mathrm{e}-5$ & $22 \%$ & $828(706 / 963)$ & $1 \mathrm{e}-5$ & $52 \%$ \\
\hline$Z_{5}$ & $2458(2301 / 2597)$ & $6 e-9$ & $100 \%$ & 1003(903/1093) & $7 \mathrm{e}-9$ & $100 \%$ \\
\hline$R_{5}$ & $2895(2523 / 3473)$ & $7 \mathrm{e}-9$ & $75 \%$ & 1684(1326/2093) & $6 e-9$ & $85 \%$ \\
\hline$H_{6,4}$ & $3978(3618 / 4308)$ & $2 \mathrm{e}-6$ & $68 \%$ & $1787(1489 / 2036)$ & $2 \mathrm{e}-6$ & $83 \%$ \\
\hline$Z_{10}$ & $16392(14235 / 17821)$ & $2 \mathrm{e}-8$ & $100 \%$ & $4032(3689 / 4809)$ & $2 \mathrm{e}-8$ & $100 \%$ \\
\hline$R_{10}$ & $19139(16844 / 22416)$ & $2 \mathrm{e}-8$ & $78 \%$ & $9037(6701 / 12879)$ & $2 \mathrm{e}-8$ & $85 \%$ \\
\hline
\end{tabular}

CRTS and DOPE in the original papers $[2,7]$.

The results of the two versions of DTS method, $\mathrm{DTS}_{N M S}$ and $\mathrm{DTS}_{A P S}$, are reported in Table 3. These results represent the average number of function evaluations (Av. $f$-evals.) with minimum and maximum numbers in parentheses, the average errors (Av. Error) and the success rates (Suc.) for each function. The average number of function evaluations and the average error only relate to successful trials. The results shown in Table 3 reveal that the performance of DTS $A P S$ is consistently better than $\mathrm{DTS}_{N M S}$ in terms of function evaluations and the ability of obtaining global minima. Moreover, it seems that DTS $\mathrm{DMS}_{N}$ suffers from the curse of dimensionality as is seen from the Av. $f$-evals. for higher dimensional problems.

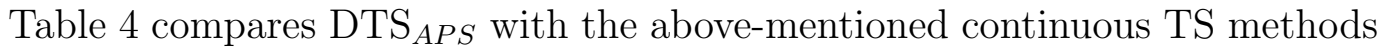
in terms of the average number of function evaluations. The results of CRTS, ECTS and DOPE methods are taken from their original papers $[2,3,7]$. The 
percentages in parentheses represent the success rates of reaching global minima. The quality of the computed solutions by those methods except the CRTS method is shown in Table 5, where the errors are measured in terms of function values at the computed and exact solutions. The quality of the produced solutions by the CRTS method is not stated clearly in [2], but it is only said that the statistical error on the CRTS is about 3\%. Before judging the comparison of these method, some remarks are made in regard to the reported success rates of ECTS and the termination condition of DOPE.

- The ECTS method uses condition (9) to test the success of a trial [3]. However, the results marked by $\left({ }^{\circledast}\right)$ in Tables 4 and 5 seem to contain some inconsistencies. In fact, from condition (9), the average errors for functions $R_{2}, R_{5}$ and $Z_{5}$ must be less than $10^{-6}$ because $f^{*}=0$ for all these functions. However, in Table 5 , they are reported to be greater than $10^{-6}$. For instance, the average error for function $R_{5}$ in Table 5 is 0.08 , which means that there are some trials that did not satisfy condition (9). Nevertheless, the rate of success is reported to be $100 \%$. Moreover, the results of ECTS for functions $R C, E S, G P, H_{3,4}$ and $H_{6,4}$ in Tables 4 and 5 also contain similar inconsistencies.

- According to [7], DOPE is terminated when either a maximum number of function evaluations is reached or the global minimum (if it is a priori known) is found. Since the information on global minima is not available in practice, we did not use the latter termination condition in our numerical experiments. This termination condition may explain the extremely small number of function evaluations of DOPE for some test functions.

From these remarks, the comparisons of the DTS methods with the ECTS and DOPE methods do not seem to yield a definitive fair answer. However, in terms of the quality of computed solutions, the DTS ${ }_{A P S}$ algorithm seems to outperform ECTS and DOPE as shown in Table 5. Moreover, the DTS $A P S$ algorithm seems to outperform ECTS in terms of the number of function evaluations for functions in Set A. However, the drawback we have noticed on the DTS algorithms is its deterioration in high dimensional problems $(n>30)$. Actually, this can be expected since the search in the DTS algorithms is mainly controlled by direct search methods and it has been shown, for instance, in [18] that the latter methods deteriorate with the increase of the dimension, i.e., suffer from the curse of dimensionality. To show the limit of deterioration of the DTS performance with the dimensionality, we report some results for high dimensional problems. The results have been obtained by running the Matlab code of $\mathrm{DTS}_{A P S}$, with the parameter setting given in Subsection 5.1, on Pentium 2.8-GHz machine. For Rosenbrock $R_{50}$ function, the $\mathrm{DTS}_{A P S}$ algorithm converged to a point close to the global minimum with function value $4.46 \times 10^{-7}$ using 510, 505 function evaluations in $1085 \mathrm{CPU}$ seconds. For Zakharov $Z_{50}$ and Rosenbrock $R_{100}$ functions, the DTS $A P S$ algorithm obtained points not so close to the global minimum at distances 1.404 and 4.1057 with 
Table 4

Average numbers of function evaluations for continuous TS methods.

\begin{tabular}{|c|c|c|c|c|c|}
\hline \multirow[b]{2}{*}{$f$} & \multirow[t]{2}{*}{$\mathrm{DTS}_{A P S}$} & \multirow[t]{2}{*}{ ECTS } & \multirow[t]{2}{*}{ DOPE } & \multicolumn{2}{|c|}{ CRTS } \\
\hline & & & & $\mathrm{CRTS}_{A v e}$ & $\mathrm{CRTS}_{\text {Min }}$ \\
\hline$R C$ & 212 & $245^{\circledast}$ & 31 & 38 & 41 \\
\hline$E S$ & $223(82 \%)$ & $1284^{\circledast}$ & 290 & - & - \\
\hline$G P$ & 230 & $231^{\circledast}$ & 248 & 248 & 171 \\
\hline$S H$ & $274(92 \%)$ & 370 & 466 & - & - \\
\hline$Z_{2}$ & 201 & 195 & 81 & - & - \\
\hline$R_{2}$ & 254 & $480^{\circledast}$ & 692 & - & - \\
\hline$D J$ & 446 & 338 & 131 & - & - \\
\hline$H_{3,4}$ & 438 & $548^{\circledast}$ & 135 & 513 & 609 \\
\hline$S_{4,5}$ & $819(75 \%)$ & $825(75 \%)$ & - & 812 & 664 \\
\hline$S_{4,7}$ & $812(65 \%)$ & $910(80 \%)$ & - & 960 & 871 \\
\hline$S_{4,10}$ & $828(52 \%)$ & $898(75 \%)$ & - & 921 & 693 \\
\hline$Z_{5}$ & 1003 & $2254^{\circledast}$ & 424 & - & - \\
\hline$R_{5}$ & $1684(85 \%)$ & $2142^{\circledast}$ & 2512 & - & - \\
\hline$H_{6,4}$ & $1787(83 \%)$ & $1520^{\circledast}$ & 421 & 750 & 1245 \\
\hline$Z_{10}$ & 4032 & 4630 & 8695 & - & - \\
\hline$R_{10}$ & $9037(85 \%)$ & $15720(85 \%)$ & 5133 & - & - \\
\hline
\end{tabular}

function values 1.972 and 4.106 using 177, 125 and 3,202, 879 function evaluations in 1,043 and 15,270 CPU seconds, respectively. These results of $Z_{50}$ and $R_{100}$ are the best among 5 runs. For Zakharov $Z_{100}$, the DTS $A P S$ algorithm failed to obtain a point near the global minimum by 5 runs using the same setting of parameters. As far as the results in Table 4 common to all methods are concerned, CRTS may be considered the best among the continuous TS methods in terms of the ability of obtaining global minima and the number of function evaluations.

\subsubsection{The performance of DTS $S_{A P S}$ against other metaheuristics}

The performance of $\mathrm{DTS}_{A P S}$ is compared with other metaheuristics using the test functions listed as Set B in Table 6 [19]. We choose two other metaheuristics proposed for the continuous optimization problem; Genetic algorithm for numerical optimization of constrained problems (Genocop III) [23], and Scatter Search (SS) [19]. We define the optimality gap (GAP) [19] as the quantity 
Table 5

Average errors for continuous TS methods.

\begin{tabular}{|c|c|c|c|c|c|c|c|}
\hline$f$ & $\mathrm{DTS}_{A P S}$ & ECTS & DOPE & $f$ & $\mathrm{DTS}_{A P S}$ & ECTS & DOPE \\
\hline$R C$ & $4 \mathrm{e}-7$ & $5 \mathrm{e}-2^{\circledast}$ & $5 e-2$ & $S_{4,5}$ & $3 \mathrm{e}-7$ & $1 \mathrm{e}-2$ & - \\
\hline$E S$ & $4 e-9$ & $1 \mathrm{e}-2^{\circledast}$ & $1 \mathrm{e}-2$ & $S_{4,7}$ & $4 e-5$ & $1 \mathrm{e}-2$ & - \\
\hline$G P$ & $5 e-9$ & $2 \mathrm{e}-3^{\circledast}$ & $2 \mathrm{e}-3$ & $S_{4,10}$ & $1 \mathrm{e}-5$ & $1 \mathrm{e}-2$ & - \\
\hline$S H$ & $9 \mathrm{e}-6$ & $1 e^{-}-3$ & $1 e^{-}-3$ & $Z_{5}$ & $7 \mathrm{e}-9$ & $4 \mathrm{e}-6^{\circledast}$ & $4 e-6$ \\
\hline$Z_{2}$ & $5 \mathrm{e}-9$ & $2 \mathrm{e}-7$ & $2 \mathrm{e}-7$ & $R_{5}$ & $6 e-9$ & $8 \mathrm{e}-2^{\circledast}$ & $8 \mathrm{e}-2$ \\
\hline$R_{2}$ & $5 \mathrm{e}-9$ & $2 \mathrm{e}-2^{\circledast}$ & $2 \mathrm{e}-2$ & $H_{6,4}$ & $2 \mathrm{e}-6$ & $5 \mathrm{e}-2^{\circledast}$ & $5 \mathrm{e}-2$ \\
\hline$D J$ & $5 e-9$ & $3 e-8$ & $3 e-8$ & $Z_{10}$ & $2 \mathrm{e}-8$ & $2 \mathrm{e}-7$ & $2 \mathrm{e}-2$ \\
\hline$H_{3,4}$ & $2 \mathrm{e}-6$ & $9 \mathrm{e}-2^{\circledast}$ & $9 \mathrm{e}-2$ & $R_{10}$ & $2 \mathrm{e}-8$ & $2 \mathrm{e}-2$ & $2 \mathrm{e}-7$ \\
\hline
\end{tabular}

on the left-hand side of (8). Table 7 shows the average GAP for all 40 test functions in Set B. In Table 7, the figures related to Genocop III and SS are taken from [19] and represent the average GAP for all test functions in Set B at intermediate stages during the search. Since the DTS $A P S$ algorithm consists of two complementary parts (Exploration-Diversification Search and Intensification Search), its results in Table 7 are the average GAP for all test functions in Set B obtained by running the DTS $A P S$ code 7 times for each test function with the termination condition that the number of function evaluations exceeds 100, 500, 1000, 5000, 10000, 20000 and 50000, respectively. Since Genocop III has a bad performance on the test function No. $23\left(S C_{6}\right)$, the results excluding this function are also included.

According to the results in Table 7, the performance of $\mathrm{DTS}_{A P S}$ is generally better than Genocop III and SS when the number of function evaluations is greater than 1000. However, in the early stage of computations, SS performs better than $\mathrm{DTS}_{A P S}$. This can be expected since $\mathrm{DTS}_{A P S}$ is a point-to-point search method while SS is a population-based search method. So, DTS ${ }_{A P S}$ may need more iterations, and therefore more function evaluations, to explore the search space well especially for high dimensional functions $(n \geq 6)$.

Since the data related to Genocop III and SS in Fig. 5 are taken from [19], we also made the successful trial test [19] for the DTS $_{A P S}$ results in our experiments. We say that a method approximately finds an optimal solution if

$$
G A P \leq \begin{cases}0.001, & \text { if } f^{*} \neq 0 \\ 0.001 f^{*}, & \text { otherewise }\end{cases}
$$

The graphs in Fig. 5 show the number of test functions from Set B that were 
Table 6

Test functions (Set B).

\begin{tabular}{llllllll}
\hline No. & $f$ & Function name & $n$ & No. & $f$ & Function name & $n$ \\
\hline 1 & $R C$ & Branin RCOS & 2 & 21 & $P S_{8,18,44,114}$ & Power Sum & 4 \\
2 & $B_{2}$ & Bohachevsky & 2 & 22 & $H_{6,4}$ & Hartmann & 6 \\
3 & $E S$ & Easom & 2 & 23 & $S C_{6}$ & Schwefel & 6 \\
4 & $G P$ & Goldstein\&Price & 2 & 24 & $T_{6}$ & Trid & 6 \\
5 & $S H$ & Shubert & 2 & 25 & $T_{10}$ & Trid & 10 \\
6 & $B L$ & Beale & 2 & 26 & $R T_{10}$ & Rastrigin & 10 \\
7 & $B O$ & Booth & 2 & 27 & $G_{10}$ & Griewank & 10 \\
8 & $M T$ & Matyas & 2 & 28 & $S S_{10}$ & Sum Squares & 10 \\
9 & $H M$ & Hump & 2 & 29 & $R_{10}$ & Rosenbrock & 10 \\
10 & $S C_{2}$ & Schwefel & 2 & 30 & $Z_{10}$ & Zakharov & 10 \\
11 & $R_{2}$ & Rosenbrock & 2 & 31 & $R T_{20}$ & Rastrigin & 20 \\
12 & $Z_{2}$ & Zakharov & 2 & 32 & $G_{20}$ & Griewank & 20 \\
13 & $D J$ & De Joung & 3 & 33 & $S S_{20}$ & Sum Squares & 20 \\
14 & $H_{3,4}$ & Hartmann & 3 & 34 & $R_{20}$ & Rosenbrock & 20 \\
15 & $C V$ & Colville & 4 & 35 & $Z_{20}$ & Zakharov & 20 \\
16 & $S_{4,5}$ & Shekel & 4 & 36 & $P W_{24}$ & Powell & 24 \\
17 & $S_{4,7}$ & Shekel & 4 & 37 & $D P_{25}$ & Dixon\&Price & 25 \\
18 & $S_{4,10}$ & Shekel & 4 & 38 & $L_{30}$ & Levy & 30 \\
19 & $P_{4,0.5}$ & Perm & 4 & 39 & $S R_{30}$ & Sphere & 30 \\
20 & $P_{4,0.5}^{0}$ & Perm & 4 & 40 & $A K_{30}$ & Ackley & 30 \\
\hline & & & & & & & \\
\hline
\end{tabular}

Table 7

Average optimality gap values.

\begin{tabular}{lrrrrrrr}
\hline$f$-evals. & \multicolumn{1}{l}{100} & \multicolumn{1}{l}{500} & \multicolumn{1}{l}{1000} & 5000 & 10000 & 20000 & 50000 \\
\hline Genocop III $^{1}$ & $5.37 \mathrm{E}+25$ & $2.39 \mathrm{E}+17$ & $1.13 \mathrm{E}+14$ & 636.37 & 399.52 & 320.84 & 313.34 \\
Genocop III $^{2}$ & 1335.45 & 611.30 & 379.03 & 335.81 & 328.66 & 324.72 & 321.20 \\
Scatter Search & 134.45 & 26.34 & 14.66 & 4.96 & 3.60 & 3.52 & 3.46 \\
DTS $_{A P S}$ & $5.04 \mathrm{E}+04$ & 43.06 & 24.26 & 4.22 & 1.80 & 1.70 & 1.29 \\
\hline
\end{tabular}

${ }^{1}$ Average values for all test functions.

${ }^{2}$ Average values for all test functions except function 23 . 


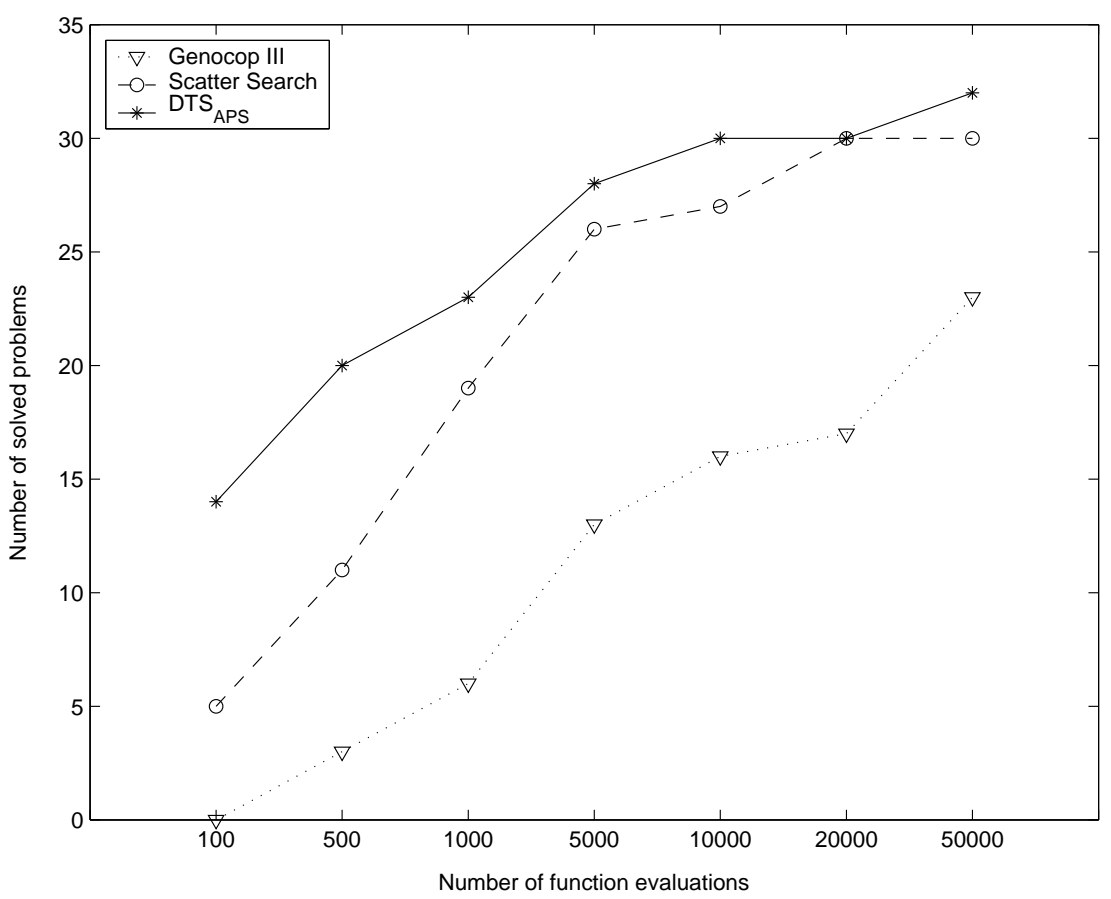

Fig. 5. Number of solved problems.

approximately solved by each method. Fig. 5 shows that DTS $_{A P S}$ could approximately find global minima for 14 test functions within only 100 function evaluations. Actually, the dimensions of these 14 test functions are less than or equal to 6 . Fig. 5 also show that $\mathrm{DTS}_{A P S}$ generally outperforms the other two methods, Genocop III and SS.

\section{Conclusion}

In this paper, we have presented a continuous TS method called Directed Tabu Search (DTS) method. First, neighborhood-local search strategies are introduced to provide more powerful search procedures to generate trial moves. A new pattern search procedure and the NM method are used to construct these neighborhood-local search strategies. Moreover, new memory elements called $T R$, Semi- $T R$ and multi-ranked $T L$ are applied to compose anti-cycling rules and to escape from local minima. Finally, a diversification scheme based on the memory element $V R L$ is introduced to explore broad areas of the solution space. The numerical results show the promise of the proposed method. 
The authors thank Rafael Marti and Manuel Laguna for providing us the data of SS and Genocop III used in Fig. 5.

\section{A Test Functions}

\section{$\left(A K_{n}\right)$ Ackley Function}

Definition: $A K_{n}(\mathbf{x})=20+e-20 e^{-\frac{1}{5} \sqrt{\frac{1}{n} \sum_{i=1}^{n} x_{i}^{2}}}-e^{-\frac{1}{n} \sum_{i=1}^{n} \cos \left(2 \pi x_{i}\right)}$.

Search space: $-15 \leq x_{i} \leq 30, i=1,2, \ldots, n$.

Global minimum: $\mathrm{x}^{*}=(0, \ldots, 0) ; A K_{n}\left(\mathrm{x}^{*}\right)=0$.

\section{$\left(B_{2}\right)$ Bohachevsky Function}

Definition: $B_{2}(\mathbf{x})=x_{1}^{2}+2 x_{2}^{2}-0.3 \cos \left(3 \pi x_{1}\right)-0.4 \cos \left(4 \pi x_{2}\right)+0.7$.

Search space: $-50 \leq x_{i} \leq 100, i=1,2$.

Global minimum: $\mathbf{x}^{*}=(0,0) ; B_{2}\left(\mathbf{x}^{*}\right)=0$.

\section{$(B L)$ Beale Function}

Definition: $B L(\mathbf{x})=\left(1.5-x_{1}+x_{1} x_{2}\right)^{2}+\left(2.25-x_{1}+x_{1} x_{2}^{2}\right)^{2}+\left(2.625-x_{1}+x_{1} x_{2}^{3}\right)^{2}$.

Search space: $-4.5 \leq x_{i} \leq 4.5, i=1,2$.

Global minimum: $\mathbf{x}^{*}=(3,0.5) ; B L\left(\mathbf{x}^{*}\right)=0$.

\section{(BO) Booth Function}

Definition: $B O(\mathbf{x})=\left(x_{1}+2 x_{2}-7\right)^{2}+\left(2 x_{1}+x_{2}-5\right)^{2}$,

Search space: $-10 \leq x_{i} \leq 10, i=1,2$

Global minimum: $\mathbf{x}^{*}=(1,3) ; B O\left(\mathbf{x}^{*}\right)=0$.

\section{$(C V)$ Colville Function}

Definition: $C V(\mathbf{x})=100\left(x_{1}^{2}-x_{2}\right)^{2}+\left(x_{1}-1\right)^{2}+\left(x_{3}-1\right)^{2}+90\left(x_{3}^{2}-x_{4}\right)^{2}+$ $10.1\left(\left(x_{2}-1\right)^{2}+\left(x_{4}-1\right)^{2}\right)+19.8\left(x_{2}-1\right)\left(x_{4}-1\right)$.

Search space: $-10 \leq x_{i} \leq 10, i=1, \ldots, 4$.

Global minimum: $\mathbf{x}^{*}=(1,1,1,1) ; C V\left(\mathbf{x}^{*}\right)=0$.

\section{$(D J)$ De Joung Function}

Definition: $D J(\mathbf{x})=x_{1}^{2}+x_{2}^{2}+x_{3}^{2}$.

Search space: $-2.56 \leq x_{i} \leq 5.12, i=1,2,3$.

Global minimum: $\mathbf{x}^{*}=(0,0,0) ; \operatorname{DJ}\left(\mathbf{x}^{*}\right)=0$.

\section{$\left(D P_{n}\right)$ Dixon\&Price Function}

Definition: $D P_{n}(\mathbf{x})=\left(x_{1}-1\right)^{2}+\sum_{i=2}^{n} i\left(2 x_{i}^{2}-x_{i-1}\right)^{2}$.

Search space: $-10 \leq x_{i} \leq 10, i=1, \ldots, n$.

Global minimum: $x_{i}^{*}=2^{-\left(\frac{2^{i}-2}{2^{i}}\right)}, i=1, \ldots, n ; D P_{n}\left(\mathbf{x}^{*}\right)=0$. 


\section{$(E S)$ Easom Function}

Definition: $E S(\mathbf{x})=-\cos \left(x_{1}\right) \cos \left(x_{2}\right) \exp \left(-\left(x_{1}-\pi\right)^{2}-\left(x_{2}-\pi\right)^{2}\right)$.

Search space: $-100 \leq x_{i} \leq 100, i=1,2$.

Global minimum: $\mathbf{x}^{*}=(\pi, \pi) ; E S\left(\mathbf{x}^{*}\right)=-1$.

\section{$\left(G_{n}\right)$ Griewank Function}

Definition: $G_{n}(\mathbf{x})=\sum_{i=1}^{n} \frac{x_{i}^{2}}{4000}-\prod_{i=1}^{n} \cos \left(x_{i} / \sqrt{i}\right)+1$.

Search space: $-300 \leq x_{i} \leq 600, i=1, \ldots, n$.

Global minimum: $\mathbf{x}^{*}=(0, \ldots, 0), G_{n}\left(\mathbf{x}^{*}\right)=0$.

\section{$(G P)$ Goldstein\&Price Function}

Definition: $G P(\mathbf{x})=\left(1+\left(x_{1}+x_{2}+1\right)^{2}\left(19-14 x_{1}+13 x_{1}^{2}-14 x_{2}+6 x_{1} x_{2}+3 x_{2}^{2}\right)\right) *$ $\left(30+\left(2 x_{1}-3 x_{2}\right)^{2}\left(18-32 x_{1}+12 x_{1}^{2}-48 x_{2}-36 x_{1} x_{2}+27 x_{2}^{2}\right)\right)$.

Search space: $-2 \leq x_{i} \leq 2, i=1,2$.

Global minimum: $\mathbf{x}^{*}=(0,-1) ; G P\left(\mathbf{x}^{*}\right)=3$.

\section{$\left(H_{3,4}\right)$ Hartmann Function}

Definition: $H_{3,4}(\mathbf{x})=-\sum_{i=1}^{4} \alpha_{i} \exp \left[-\sum_{j=1}^{3} A_{i j}\left(x_{j}-P_{i j}\right)^{2}\right], \alpha=[1,1.2,3,3.2]^{T}$, $A=\left[\begin{array}{lll}3.0 & 10 & 30 \\ 0.1 & 10 & 35 \\ 3.0 & 10 & 30 \\ 0.1 & 10 & 35\end{array}\right], P=10^{-4}\left[\begin{array}{ccc}6890 & 1170 & 2673 \\ 4699 & 4387 & 7470 \\ 1091 & 8732 & 5547 \\ 381 & 5743 & 8828\end{array}\right]$.

Search space: $0 \leq x_{i} \leq 1, i=1,2,3$.

Global minimum: $\mathbf{x}^{*}=(0.114614,0.555649,0.852547) ; H_{3,4}\left(\mathbf{x}^{*}\right)=-3.86278$.

\section{$\left(H_{6,4}\right)$ Hartmann Function}

Definition: $H_{6,4}(\mathbf{x})=-\sum_{i=1}^{4} \alpha_{i} \exp \left[-\sum_{j=1}^{6} B_{i j}\left(x_{j}-Q_{i j}\right)^{2}\right], \alpha=[1,1.2,3,3.2]^{T}$,

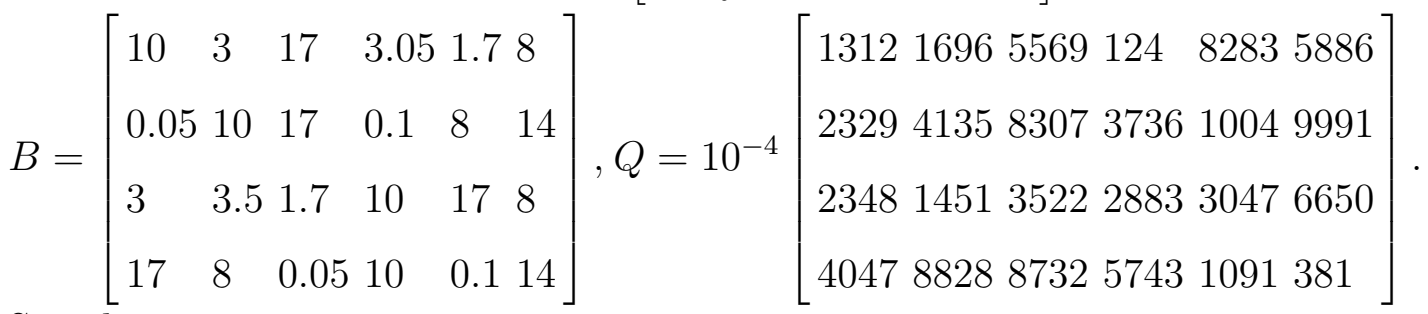

Search space: $0 \leq x_{i} \leq 1, i=1, \ldots, 6$.

Global minimum: $\mathbf{x}^{*}=(0.201690,0.150011,0.476874,0.275332,0.311652,0.657300)$; $H_{6,4}\left(\mathbf{x}^{*}\right)=-3.32237$.

\section{( $H M)$ Hump Function}

Definition: $H M(\mathbf{x})=4 x_{1}^{2}-2.1 x_{1}^{4}+\frac{1}{3} x_{1}^{6}+x_{1} x_{2}-4 x_{2}^{2}+4 x_{2}^{4}$.

Search space: $-5 \leq x_{i} \leq 5, i=1,2$.

Global minimum: $\mathbf{x}^{*}=(0.0898,-0.7126),(-0.0898,0.7126) ; H M\left(\mathbf{x}^{*}\right)=0$. 


\section{$\left(L_{n}\right)$ Levy Function}

Definition: $L_{n}(\mathbf{x})=\sin ^{2}\left(\pi y_{1}\right)+\sum_{i=1}^{n-1}\left[\left(y_{i}-1\right)^{2}\left(1+10 \sin ^{2}\left(\pi y_{i}+1\right)\right)\right]$

$+\left(y_{n}-1\right)^{2}\left(1+10 \sin ^{2}\left(2 \pi y_{n}\right)\right), y_{i}=1+\frac{x_{i}-1}{4}, i=1, \ldots, n$.

Search space: $-10 \leq x_{i} \leq 10, i=1, \ldots, n$.

Global minimum: $\mathbf{x}^{*}=(1, \ldots, 1) ; L_{n}\left(\mathbf{x}^{*}\right)=0$.

\section{(MT) Matyas Function}

Definition: $M T(\mathbf{x})=0.26\left(x_{1}^{2}+x_{2}^{2}\right)-0.48 x_{1} x_{2}$.

Search space: $-5 \leq x_{i} \leq 10, i=1,2$.

Global minimum: $\mathbf{x}^{*}=(0,0) ; M T\left(\mathbf{x}^{*}\right)=0$.

\section{$\left(P_{n, \beta}\right)$ Perm Function}

Definition: $P_{n, \beta}(\mathbf{x})=\sum_{k=1}^{n}\left[\sum_{i=1}^{n}\left(i^{k}+\beta\right)\left(\left(x_{i} / i\right)^{k}-1\right)\right]^{2}$.

Search space: $-n \leq x_{i} \leq n, i=1, \ldots, n$.

Global minimum: $\mathbf{x}^{*}=(1,2, \ldots, n) ; P_{n, \beta}\left(\mathbf{x}^{*}\right)=0$.

\section{$\left(P_{n, \beta}^{0}\right)$ Perm Function}

Definition: $P_{n, \beta}^{0}(\mathbf{x})=\sum_{k=1}^{n}\left[\sum_{i=1}^{n}(i+\beta)\left(x_{i}^{k}-(1 / i)^{k}\right)\right]^{2}$.

Search space: $-n \leq x_{i} \leq n, i=1, \ldots, n$.

Global minimum: $\mathbf{x}^{*}=\left(1, \frac{1}{2}, \ldots, \frac{1}{n}\right) ; P_{n, \beta}^{0}\left(\mathbf{x}^{*}\right)=0$.

\section{$\left(P S_{b_{1}, \ldots, b_{n}}\right)$ Power Sum Function}

Definition: $P S_{b_{1}, \ldots, b_{n}}(\mathbf{x})=\sum_{k=1}^{n}\left[\left(\sum_{i=1}^{n} x_{i}^{k}\right)-b_{k}\right]^{2}$.

Search space: $0 \leq x_{i} \leq n, i=1, \ldots, n$.

Global minimum for $P S_{8,18,44,114}(\mathbf{x}): \mathbf{x}^{*}=(1,2,2,3) ; P S_{8,18,44,114}\left(\mathbf{x}^{*}\right)=0$.

$\left(P W_{n}\right)$ Powell Function

Definition: $P W_{n}(\mathbf{x})=\sum_{i=1}^{n / 4}\left(x_{4 i-3}+10 x_{4 i-2}\right)^{2}+5\left(x_{4 i-1}-x_{4 i}\right)^{2}+\left(x_{4 i-2}-x_{4 i-1}\right)^{4}+$ $10\left(x_{4 i-3}-x_{4 i}\right)^{4}$.

Search space: $-4 \leq x_{i} \leq 5, i=1, \ldots, n$.

Global minimum: $\mathbf{x}^{*}=(3,-1,0,1,3, \ldots, 3,-1,0,1) ; P W_{n}\left(\mathbf{x}^{*}\right)=0$.

\section{$\left(R_{n}\right)$ Rosenbrock Function}

Definition: $R_{n}(\mathbf{x})=\sum_{i=1}^{n-1}\left[100\left(x_{i}^{2}-x_{i+1}\right)^{2}+\left(x_{i}-1\right)^{2}\right]$.

Search space: $-5 \leq x_{i} \leq 10, i=1,2, \ldots, n$.

Global minimum: $\mathbf{x}^{*}=(1, \ldots, 1), R_{n}\left(\mathbf{x}^{*}\right)=0$.

\section{$(R C)$ Branin RCOS Function}

Definition: $R C(\mathbf{x})=\left(x_{2}-\frac{5}{4 \pi^{2}} x_{1}^{2}+\frac{5}{\pi} x_{1}-6\right)^{2}+10\left(1-\frac{1}{8 \pi}\right) \cos \left(x_{1}\right)+10$.

Search space: $-5 \leq x_{1} \leq 10,0 \leq x_{2} \leq 15$.

Global minima: $\mathbf{x}^{*}=(-\pi, 12.275),(\pi, 2.275),(9.42478,2.475) ; R C\left(\mathbf{x}^{*}\right)=0.397887$.

\section{$\left(R T_{n}\right)$ Rastrigin Function}

Definition: $R T_{n}(\mathbf{x})=10 n+\sum_{i=1}^{n}\left(x_{i}^{2}-10 \cos \left(2 \pi x_{i}\right)\right)$. 
Search space: $-2.56 \leq x_{i} \leq 5.12, i=1, \ldots, n$.

Global minimum: $\mathbf{x}^{*}=(0, \ldots, 0), R T_{n}\left(\mathbf{x}^{*}\right)=0$.

\section{$\left(S_{4, m}\right)$ Shekel Function}

Definition: $S_{4, m}(\mathbf{x})=-\sum_{j=1}^{m}\left[\sum_{i=1}^{4}\left(x_{i}-C_{i j}\right)^{2}+\beta_{j}\right]^{-1}, \beta=\frac{1}{10}[1,2,2,4,4,6,3,7,5,5]^{T}$, $C=\left[\begin{array}{llllllllllll}4.0 & 1.0 & 8.0 & 6.0 & 3.0 & 2.0 & 5.0 & 8.0 & 6.0 & 7.0 \\ 4.0 & 1.0 & 8.0 & 6.0 & 7.0 & 9.0 & 5.0 & 1.0 & 2.0 & 3.6 \\ 4.0 & 1.0 & 8.0 & 6.0 & 3.0 & 2.0 & 3.0 & 8.0 & 6.0 & 7.0 \\ 4.0 & 1.0 & 8.0 & 6.0 & 7.0 & 9.0 & 3.0 & 1.0 & 2.0 & 3.6\end{array}\right]$.

Search space: $0 \leq x_{i} \leq 10, i=1, \ldots, 4$.

Global minima: $\mathbf{x}^{*}=(4,4,4,4) ; S_{4,5}\left(\mathbf{x}^{*}\right)=-10.1532, S_{4,7}\left(\mathbf{x}^{*}\right)=-10.4029$ and $S_{4,10}\left(\mathbf{x}^{*}\right)=-10.5364$.

\section{$\left(S C_{n}\right)$ Schwefel Function}

Definition: $S C_{n}(\mathbf{x})=418.9829 n-\sum_{i=1}^{n}\left(x_{i} \sin \sqrt{\left|x_{i}\right|}\right)$.

Search space: $-500 \leq x_{i} \leq 500, i=1,2, \ldots, n$.

Global minimum: $\mathbf{x}^{*}=(1, \ldots, 1), S C_{n}\left(\mathbf{x}^{*}\right)=0$.

\section{$(S H)$ Shubert Function}

Definition: $S H(\mathbf{x})=\left(\sum_{i=1}^{5} i \cos \left((i+1) x_{1}+i\right)\right)\left(\sum_{i=1}^{5} i \cos \left((i+1) x_{2}+i\right)\right)$,

Search space: $-10 \leq x_{i} \leq 10, i=1,2$

Global minima: 18 global minima and $S H\left(\mathbf{x}^{*}\right)=-186.7309$.

$\left(S R_{n}\right)$ Sphere Function

Definition: $S R_{n}(\mathbf{x})=\sum_{i=1}^{n} x_{i}^{2}$,

Search space: $-2.56 \leq x_{i} \leq 5.12, i=1, \ldots, n$

Global minimum: $\mathbf{x}^{*}=(0, \ldots, 0), S R_{n}\left(\mathbf{x}^{*}\right)=0$.

\section{$\left(S S_{n}\right)$ Sum Squares Function}

Definition: $S S_{n}(\mathbf{x})=\sum_{i=1}^{n} i x_{i}^{2}$,

Search space: $-5 \leq x_{i} \leq 10, i=1, \ldots, n$

Global minimum: $\mathbf{x}^{*}=(0, \ldots, 0), S S_{n}\left(\mathbf{x}^{*}\right)=0$.

\section{$\left(T_{n}\right)$ Trid Function}

Definition: $T_{n}(\mathbf{x})=\sum_{i=1}^{n}\left(x_{i}-1\right)^{2}-\sum_{i=2}^{n} x_{i} x_{i-1}$,

Search space: $-n^{2} \leq x_{i} \leq n^{2}, i=1, \ldots, n$

Global minima: a) $n=6, x_{i}^{*}=i(7-i), i=1, \ldots, n, T_{n}\left(\mathbf{x}^{*}\right)=-50$,

b) $n=10, x_{i}^{*}=i(11-i), i=1, \ldots, n, T_{n}\left(\mathbf{x}^{*}\right)=-210$,

\section{$\left(Z_{n}\right)$ Zakharov Function}

Definition: $Z_{n}(\mathbf{x})=\sum_{i=1}^{n} x_{i}^{2}+\left(\sum_{i=1}^{n} 0.5 i x_{i}\right)^{2}+\left(\sum_{i=1}^{n} 0.5 i x_{i}\right)^{4}$.

Search space: $-5 \leq x_{i} \leq 10, i=1,2, \ldots, n$

Global minimum: $\mathbf{x}^{*}=(0, \ldots, 0), Z_{n}\left(\mathbf{x}^{*}\right)=0$. 


\section{References}

[1] K.S. Al-Sultan, M. A. Al-Fawzan, A tabu search Hooke and Jeeves algorithm for unconstrained optimization, European Journal of Operational Research 103 (1997) 198-208.

[2] R. Battiti, G. Tecchiolli, The continuous reactive tabu search: Blending combinatorial optimization and stochastic search for global optimization, Annals of Operations Research 63 (1996) 153-188.

[3] R. Chelouah, P. Siarry, Tabu search applied to global optimization, European Journal of Operational Research 123 (2000) 256-270.

[4] D. Cvijovic, J. Klinowski, Taboo search: An approach to the multiple minima problem, Science 667 (1995) 664-666.

[5] D. Cvijovic, J. Klinowski, Taboo search: An approach to the multiple-minima problem for continuous functions, in: P. M. Pardalos and H. E. Romeijn (Eds.), Handbook of Global Optimization, Kluwer Academic Publishers, Boston, MA, 2002, pp. 387-406.

[6] C. A. Floudas, P. M. Pardalos, C. S. Adjiman, W. R. Esposito, Z. Gumus, S. T. Harding, J. L. Klepeis, C. A. Meyer, C. A. Schweiger (Eds.), Handbook of Test Problems for Local and Global Optimization, Kluwer Academic Publishers, Boston, MA, 1999.

[7] F. Franze, N. Speciale, A tabu-search-based algorithm for continuous multiminima problems, International Journal for Numerical Engineering 50 (2001) 665-680.

[8] F. Glover, Tabu search-Part I, ORSA Journal on Computing 1 (1989) 190-206.

[9] F. Glover, Tabu search-Part II, ORSA Journal on Computing 2 (1990) 4-32.

[10] F. Glover, M. Laguna, Tabu Search, Kluwer Academic Publishers, MA, USA, 1997.

[11] F. Glover, E. Taillard, D. Werra, A user's guide to tabu search, Annals of Operations Research 41 (1993) 3-28.

[12] A. Hedar, M. Fukushima, Hybrid simulated annealing and direct search method for nonlinear unconstrained global optimization. Optimization Methods and Software 17 (2002) 891-912.

[13] A. Hedar, M. Fukushima, Minimizing multimodal functions by simplex coding genetic algorithm, Optimization Methods and Software 18 (2003) 265-282.

[14] A. Hedar, M. Fukushima, Heuristic pattern search and its hybridization with simulated annealing for nonlinear global optimization, Optimization Methods and Software 19 (2004) 291-308.

[15] N. Hu, Tabu search method with random moves for globally optimal design, International Journal for Numerical Engineering 35 (1992) 1055-1070. 
[16] C. T. Kelley, Detection and remediation of stagnation in the Nelder-Mead algorithm using a sufficient decrease condition, SIAM Journal on Optimization 10 (1999) 43-55.

[17] C. T. Kelley, Iterative Methods for Optimization, Frontiers Appl. Math. 18, SIAM, Philadelphia, PA, 1999.

[18] T.G. Kolda, R.M. Lewis, V. Torczon, Optimization by direct search: New perspectives on some classical and modern methods, SIAM Review 45 (2003) $385-482$.

[19] M. Laguna, R. Martí, Experimental Testing of Advanced Scatter Search Designs for Global Optimization of Multimodal Functions, Technical Report, University of Colorado at Boulder, 2002.

[20] M. Laguna, R. Martí, V. Campos, Intensification and diversification with elite tabu search solutions for the linear ordering problem, Computers and Operations Research 26 (1999) 1217-1230.

[21] R. Martí, Multi-Start Methods , in: F. Glover and G. Kochenberger (Eds.), Handbook of MetaHeuristics, Kluwer Academic Publishers, Boston, MA, 2002, pp. 355-368.

[22] R. Martí, J.M. Moreno, Métodos multi-arranque, Inteligencia Artificial 19 (2003) 49-60.

[23] Z. Michalewicz, G. Nazhiyath, Genocop III: A co-evolutionary algorithm for numerical optimization problems with nonlinear constraints, Proceedings of the second IEEE ICEC, Preth, Australia, 1995.

[24] J. A. Nelder, R. Mead, A simplex method for function minimization, The Computer Journal 7 (1965) 308-313.

[25] P. M. Pardalos, M. G. C. Resende (Eds.), Handbook of Applied Optimization, Oxford University Press, Oxford, 2002.

[26] C.C. Ribeiro, P. Hansen (Eds.), Essays and Surveys in Metaheuristics, Kluwer Academic Publishers, Boston, MA, 2002.

[27] F. Schoen, Two phase methods for globale optimization, in: P. M. Pardalos and H. E. Romeijn (Eds.), Handbook of Global Optimization, Kluwer Academic Publishers, Boston, MA, 2002, pp. 151-178. 